\title{
Design of the fiber optic support system and fiber bundle accelerated life test for VIRUS
}

\author{
Ian M. Soukup ${ }^{\mathrm{a}}$, Joseph H. Beno ${ }^{\mathrm{a}}$, Richard J. Hayes ${ }^{\mathrm{a}}$, James T. Heisler ${ }^{\mathrm{a}}$, Jason R. Mock ${ }^{\mathrm{a}}$, Nicholas \\ T. Mollison ${ }^{\mathrm{a}}$, John M. Good ${ }^{\mathrm{b}}$, Gary J. Hill ${ }^{\mathrm{b}}$, Brian L. Vattiat ${ }^{\mathrm{b}}$, Jeremy D. Murphy ${ }^{\mathrm{c}}$, Seth C. \\ Anderson $^{\mathrm{d}}$, Svend M. Bauer ${ }^{\mathrm{e}}$, Andreas Kelz ${ }^{\mathrm{e}}$, Martin M. Roth ${ }^{\mathrm{e}}$, and Eric P. Fahrenthold ${ }^{\mathrm{f}}$ \\ ${ }^{a}$ The University of Texas Center for Electromechanics, 1 University Station R7000, Austin, Texas \\ USA 78712 \\ ${ }^{b}$ The University of Texas McDonald Observatory, 1 University Station C1402, Austin, \\ Texas, USA 78712-0259 \\ ${ }^{\mathrm{c}}$ The University of Texas Department of Astronomy, 1 University Station C1400, Austin, Texas, \\ USA 78712-0259 \\ d904 W 22nd 1/2 St., Apt 214, Austin, Texas, USA 78712-0259 \\ eAstrophysikalisches Institute Potsdam, An der Sternwarte 16, 14482 Potsdam, Germany \\ ${ }^{\mathrm{f}}$ The University of Texas Department of Mechanical Engineering, ETC 4.136, 1 University Station \\ C2200, Austin, Texas, USA 78712-0292
}

\begin{abstract}
The quantity and length of optical fibers required for the Hobby-Eberly Telescope* Dark Energy eXperiment (HETDEX) create unique fiber handling challenges. For HETDEX", at least 33,600 fibers will transmit light from the focal surface of the telescope to an array of spectrographs making up the Visible Integral-Field Replicable Unit Spectrograph (VIRUS). Up to 96 Integral Field Unit (IFU) bundles, each containing 448 fibers, hang suspended from the telescope's moving tracker located more than 15 meters above the VIRUS instruments. A specialized mechanical system is being developed to support fiber optic assemblies onboard the telescope. The discrete behavior of 448 fibers within a conduit is also of primary concern. A life cycle test must be conducted to study fiber behavior and measure Focal Ratio Degradation (FRD) as a function of time. This paper focuses on the technical requirements and design of the HETDEX fiber optic support system, the electro-mechanical test apparatus for accelerated life testing of optical fiber assemblies. Results generated from the test will be of great interest to designers of robotic fiber handling systems for major telescopes. There is concern that friction, localized contact, entanglement, and excessive tension will be present within each IFU conduit and contribute to FRD. The test apparatus design utilizes six linear actuators to replicate the movement of the telescope over 65,000 accelerated cycles, simulating five years of actual operation.

Keywords: Center for Electromechanics, CEM, Hobby-Eberly Telescope, HET, HETDEX, University of Texas, McDonald Observatory, fiber optics, Integral Field Unit, IFU, Focal Ratio Degradation, FRD, VIRUS, accelerated life test

* The Hobby-Eberly Telescope is operated by McDonald Observatory on behalf of the University of Texas at Austin, the Pennsylvania State University, Stanford University, Ludwig-Maximillians-Universität München,and Georg-AugustUniversität, Göttingen

† R.S.: E-mail: i.soukup@cem.utexas.edu

$\$$ http://hetdex.org/
\end{abstract}

\section{INTRODUCTION}

The Hobby Eberly Telescope, designed to gather large amounts of light for spectroscopy, is undergoing a transformation to begin the search for "Dark Energy". Dark energy is a term used to describe unknown forces causing the acceleration of cosmic expansion ${ }^{1,2}$. To help define the evolution and significant properties of dark energy, the Hobby Eberly Telescope at the McDonald Observatory (MDO) was chosen to survey and map the locations of other galaxies in space. The experiment, which is estimated to be completed over 100 nights of viewing, is now referred to as HETDEX ${ }^{3}$. The fabrication and installation of 192 spectrographs called VIRUS $^{4,5}$ is the foundation of the telescope upgrade ${ }^{3}$. The 
VIRUS instruments are feed by as many as 43,008 optical fibers that initiate at the focal surface and are mechanically coupled and guided by strain reliefs in route to VIRUS ${ }^{6}$; the strain reliefs define the fiber optic support system. The fiber optic support system is instrumental in the successful operation of the HETDEX survey. This system has three primary objectives: efficiently guide fibers creating the shortest route to VIRUS, prevent fiber stress that could result in FRD, and integrate to telescope components without adding to light obscuration. Fiber management is important because studies have concluded that mechanical deformation of optical fibers can result in $\mathrm{FRD}^{7-10}$. Predicting the fiber behavior for HETDEX is very difficult due the size and complexity of the fiber system. Long fiber runs, large motion envelopes and thousands of optical fibers will likely complicate the mechanical behavior of the fibers in ways that require better understanding before full implementation on the telescope. A life cycle test will be conducted to study fiber behavior and measure focal ratio degradation as a function of time. The life cycle test will guide the detailed design of the fiber optic support system as well as provide results illustrating the effects of accelerated load histories on performance of optical fibers.

\section{FIBER OPTIC SUPPORT SYSTEM}

\subsection{System overview}

There are three points on the telescope that structurally support the IFUs. The locations of the IFU end terminations and these three points define the HETDEX optical fiber system routing from its input at the focal surface to its output at the VIRUS cabinets. The three points of mechanical coupling and structural support are referred to as strain reliefs and are identified from input to output as Strain Relief 1 (SR1), Strain Relief 2 (SR2), and Strain Relief 3 (SR3), Figure 1.

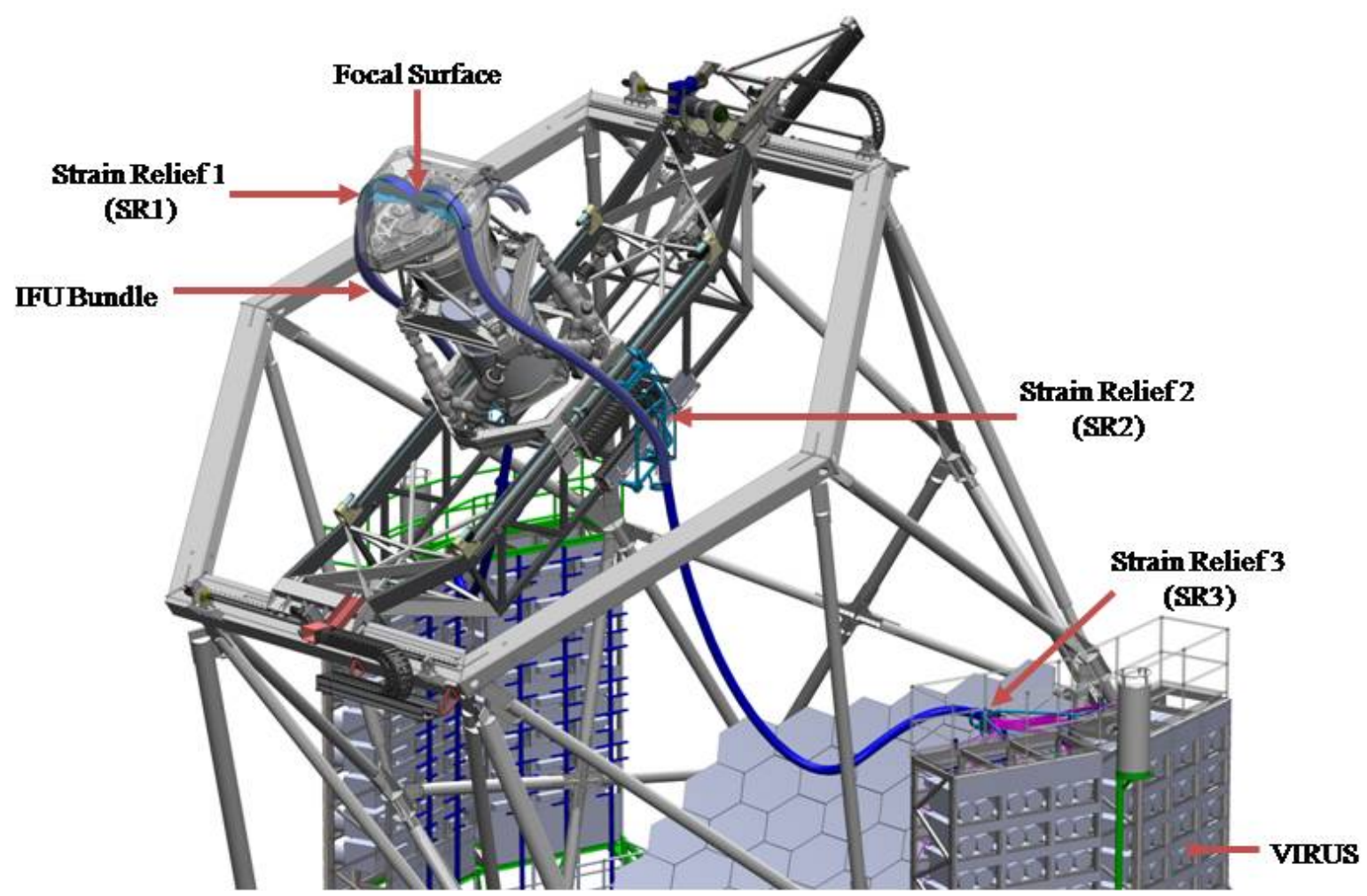

Figure 1. The HETDEX optical fiber system is illustrated above. Strain Relief 1, Strain Relief 2, and Strain Relief 3 are colored light blue. The fiber bundles routed from the focal surface to VIRUS are colored dark blue.

The HETDEX optical fiber system path consists of two runs that hang from the tracker. Each run contains up to 48 IFUs that retain a total of 21,504 optical fibers ${ }^{6}$. An IFU consists of 448 optical fibers, spiral wound aluminum conduit, and a PolyVinyl Carbonate (PVC) jacket ${ }^{11}$. Internally, Kevlar sheathing protects the fibers by reducing friction and preventing abrasion between the fibers and the aluminum conduit. The sheathing is also being used to determine the overall length of the conduit since it is dimensionally more stable along its length than the conduit. Fiber core diameter is 266 microns resulting in a $30 \%$ conduit fill factor, or the ratio of total fiber cross section area to conduit cross section $\operatorname{area}^{6}$. The 
unoccupied volume within the conduit permits differences in relative length and bend radius between conduit and fiber as each flexes during HET operation. The conduit outside diameter is $17.2 \mathrm{~mm}$ with a mass per unit length of $300 \mathrm{~g} / \mathrm{m}^{11}$. The fibers within each conduit have a mass per unit length of $80 \mathrm{~g} / \mathrm{m}^{11}$. The average IFU length is approximately $21 \mathrm{~m}$ to accommodate telescope movement and the distance between the focal surface and virus cabinets.

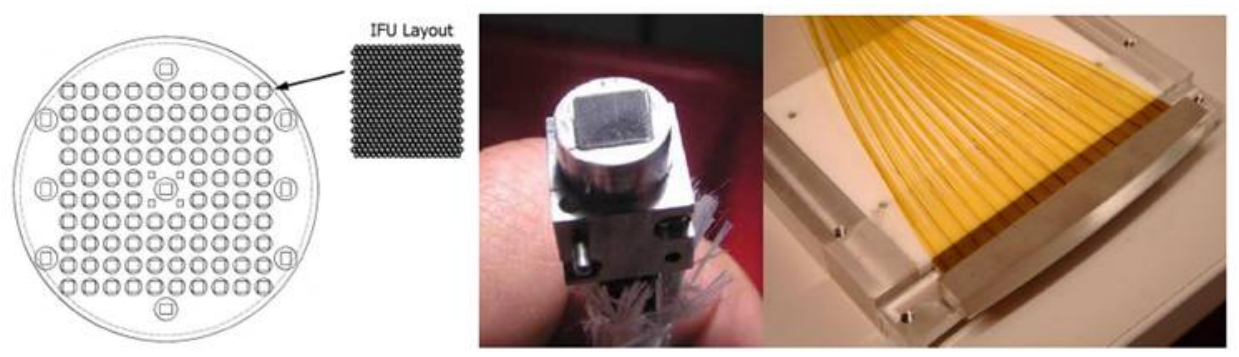

Figure 2. The image on the left is a sketch of the IFU Focal Surface Mount plate. The fiber input head and output region are displayed in the pictures to the right.

The optical fibers are coupled to the conduit at the input end through a component called the "hub". At the exit end the optical fibers exit the conduit and are terminated at the VIRUS cabinets that are located on each side of the telescope structure. Between the input and exit ends the fibers are shielded by the conduit, but are not coupled to the conduit. Three strain reliefs positioned between the IFU input and exit provide mechanical coupling to support and control the IFU conduit. The IFU's have been designed and fabricated in partnership with Astrophysikalisches Institut Potsdam (AIP). Detailed information regarding the IFUs can be located in the "Production and performance of replicable integral field units for VIRUS", reference 11.

For fiber management, preventing tight bend radii is important to prevent FRD and ultimately; fiber damage. The fiber optic support system will allow no less than a bend radius of $150 \mathrm{~mm}$ during operation. The conduit will permit a minimum bend radius of $100 \mathrm{~mm}$ during installation and handling while the individual fibers have been designed to handle bend radii less than $100 \mathrm{~mm}$ without jeopardizing optical performance.

The optical fiber system routing is summarized in Table 1. The fiber run is split into segments between each Mechanical Coupling Point (MCP) for reference throughout the paper. The behavior of fibers in each segment is listed; "static" implies that there is no relative movement between the points on either end of that segment while "dynamic" means that relative movement between the coupling points exists.

Table 1. Fiber System Layout and Mechanical Coupling

\begin{tabular}{|c|l|l|l|c|}
\hline Fiber Segment & MCP Beginning & MCP End & Behavior & Length (m) \\
\hline 1 & Focal Surface & Strain Relief 1 & Static & $<1$ \\
\hline 2 & Strain Relief 1 & Strain Relief 2 & Dynamic & 7 \\
\hline 3 & Strain Relief 2 & Strain Relief 3 & Dynamic & 9 \\
\hline 4 & Strain Relief 3 & VIRUS Cabinets & Static & 4 \\
\hline
\end{tabular}

\subsection{Strain Relief 1}

Strain Relief 1 attaches to the top of the telescope and securely clamps and guides the conduit out away from the surrounding telescope structure and equipment. SR1 isolates the input end of the fibers at the focal surface from motions of the tracker and Prime Focus Instrument Package (PFIP). This structure carries the weight of the IFUs, fibers and conduit, between the focal surface and strain relief 2, as well as a majority of the weight of the fiber from Strain Relief 2 to Strain Relief 3. A compound surface curvature is the key design feature that defines the shape of SR1. This unique shape gently redirects the IFU bundle from a normal projection at the focal surface to an unstressed free hanging state that aligns with gravity at the exit of SR1. Design for accessibility, maintenance and installation are also considerations that drove the design. The structure will position the fiber bundles away from the focal plane assembly to provide space for and access to other instrumentation. Strain Relief 1 allows the IFUs to be installed one at a time by securing each IFU individually through a multi-sectioned split clamp. 


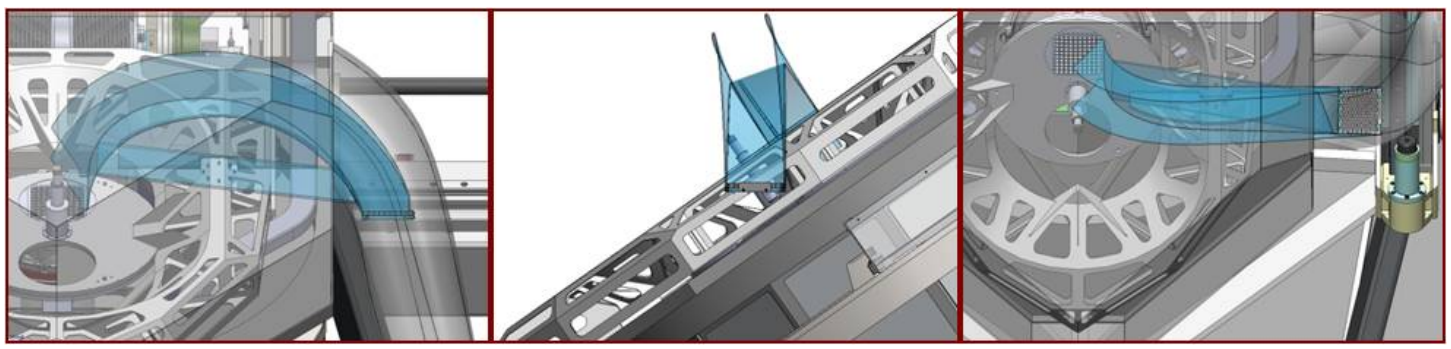

Figure 3. Strain Relief 1 is shown in blue on the telescope. The images illustrate the front view, side view, and top view from left to right. The path of the IFU bundle through SR1 is most clearly displayed in the side view.

\subsection{Strain Relief 2}

The telescope tracker moves across the primary mirror with an end-to-end travel of 4 meters in $\mathrm{X}$ and $\mathrm{Y}$ as described in papers 12,13. The fibers bundles are coupled to the $\mathrm{X}$ and $\mathrm{Y}$ axis tracker motion at Strain Relief 2. Strain Relief 2 is designed to guide and funnel the fiber bundle into an array of conduit clamps. Each IFU is clamped with rubber grommets that securely tighten around the circumference of the conduit. The grommets are retained by sectioned aluminum extrusions that apply clamping pressure through the grommets and to the conduit when multiple halves are fastened together. Each aluminum extrusion assembly retains 8 IFU's. The 6 aluminum extrusions assemblies are retained in a slotted plate that compartmentalizes the IFU bundle. Several advantages are attained by using 6 clamping assembly units as opposed to one: individual IFUs are more readily accessible, and a more evenly distributed clamping load is applied to each IFU. After passing through the clamping assemblies the IFU's are gathered by an adjustable guide ring before exiting toward Strain Relief 3. The IFU Carriage is the structure that supports the clamping assemblies. This structure attaches to the tracker bridge using a set of linear bearings that allow movement along the long axis of the bridge(Y-axis). The $\mathrm{Y}$-axis movement of the IFU carriages is mechanically coupled to the lower hexapod frame through flexible joints that convey forces only in the direction of the Y-axis. This feature helps to isolate IFU loads from the precision tracking axes of the tracker, and allows for misalignment between the bearing rails attached to the bridge and the lower hexapod frame bearings. Rail misalignment must be considered because the IFU carriage bearing rails are positioned on orthogonal surfaces of the tracker bridge, making fabrication of precise datum surfaces and rail alignment very difficult. The upper bearing rail is positioned on the side of the tracker bridge while the lower bearing rail is positioned underneath the bridge truss. This arrangement of bearing rails is favorable for minimizing impacts on obscuration of star light (vignetting) as the lower bearing rail is hidden in the shadow of the bridge structure.

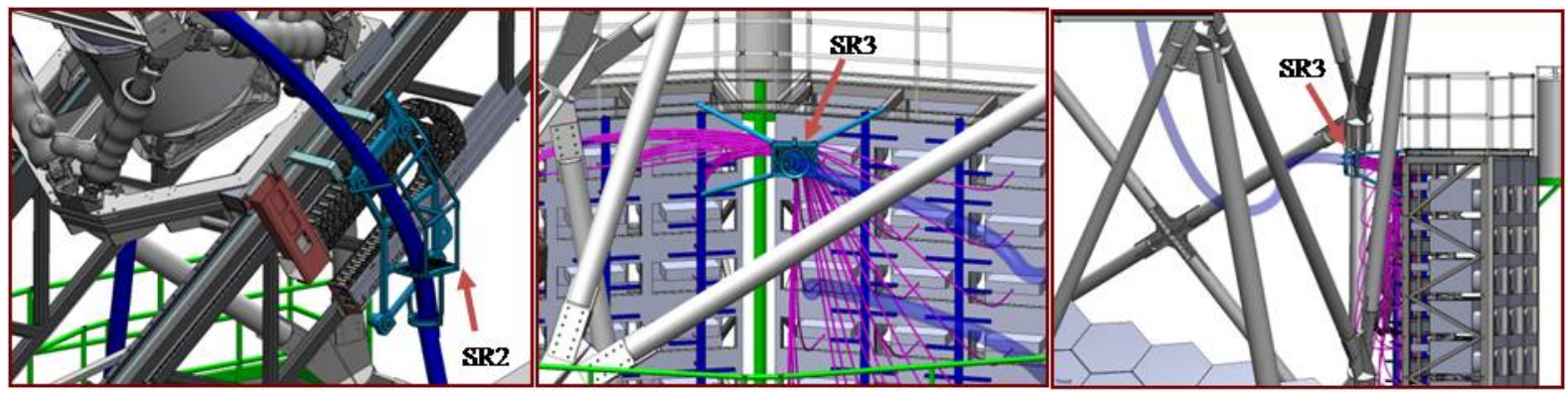

Figure 4. Strain Relief 2, carriage and clamp, is shown in the image on the left attached to the tracker lower hexapod frame.

Strain Relief 3 is illustrated in the center and right images. Both SR2 and SR3 are highlighted in light blue.

\subsection{Strain Relief 3}

The fiber bundles will either be attached to the telescope structure or to the VIRUS Support Structure (VSS) at Strain Relief 3 which supports the lower end of the IFUs just before they branch out and enter the VIRUS breakout boxes. The design of Strain Relief 3 is at the conceptual stage and will leverage lessons learned during the design of SR1 and SR2 and life cycle testing. As in SR1 and SR2 the IFU conduits will be individually supported at Strain Relief 3. Utilizing the design efforts for SR2 a very similar conduit clamping device will be deployed at this location. The conduit clamping assemblies for Strain Relief 3 will be supported by a coupling that attaches to the telescope or the VSS. The clamping assembly will be positioned between and near the top of the VIRUS towers on each side of the primary mirror. The 
alignment of the clamping assembly is defined such that a smooth transition occurs between the hanging fiber bundles from the tracker to the IFU entrance into the VIRUS breakout boxes.

\section{IFU TESTING MECHANICAL DESIGN}

\subsection{Accelerated Life Test Introduction}

The long-term performance of the fiber system is considered a risk to the HETDEX project. Optical performance of the fibers is unknown after repeated cycles of extending, contracting and flexing of the IFUs occurs during telescope tracker movement. The size and complexity of the fiber system for HETDEX is much greater than previous fiber systems deployed. Unknown problems that arise can lead to costly operational shut downs while solutions are developed.

There is specific interest in whether the bulk dynamics of the fibers under self-weighted tension and translations will impose stresses or mechanical deformations on individual fibers. Previous test and research have concluded that mechanical deformation of the optical fibers can cause $\mathrm{FRD}^{7-10}$. Due to the complexities of the fiber handling system there is concern that localized stress can develop in individual fibers. As localized stress has been shown to cause extreme cases of FRD, understanding where localized stress can develop, how it evolves with time, and what steps can be taken to mitigate localized stress is a primary driver of the life cycle tests. Detailed studies of transmission and FRD over the wavelength range of VIRUS ( 350 to $580 \mathrm{~nm}$ ) are currently being conducted at the University of Texas, Austin. Therefore, we will monitor only the relative changes in the output f-ratio of many fibers simultaneously over time, allowing us to effectively track changes in FRD with fiber bundle position. A separate lab test on the absolute transmission and FRD of the fiber bundle, conducted before and periodically during the testing cycle, will allow us to determine if any long term degradation is seen over the duration of the lifetime test.

\subsection{Test Apparatus Constraints}

Design drivers and constraints for the accelerated life test are derived from the fiber system and fiber support system that will be deployed for HETDEX. The fundamental objective is to provide a life cycle test simulating the HETDEX fiber system behavior, while continuously measuring relative changes in the output focal ratio of individual fibers over the duration of the test. The test apparatus must provide similar movements, speeds, and mechanical coupling of the fiber system, while integrating optical equipment to measure FRD. The need to replicate the routing of the fiber system on the telescope led to the decision to conduct the test within the Task B Gun Range at CEM, a unique laboratory that extends more than 45 meters underground. The depth of this facility accommodates the 15-18 meters of vertical space needed to route the fiber system, eliminating the need to fabricate an elevated structure above ground. A summary of the constraints and their drivers are listed in Table 2.

Table 2. IFU accelerated life test design constraints.

\begin{tabular}{|c|l|l|}
\hline Item & Constraint Driver & Constraint Description \\
\hline 1 & $\begin{array}{l}\text { Deploy HETDEX strain reliefs at their proposed } \\
\text { relative spatial orientation }\end{array}$ & Defines Routing (Volume) \\
\hline 2 & $\begin{array}{l}\text { Operate Test within Task B Gun Range } \\
\text { beplicate full range of telescope fiber movement }\end{array}$ & Defines Volume \\
\hline 3 & Restrain 48 IFU Conduits & Defines Force \\
\hline 4 & $\begin{array}{l}\text { Perform 5 year projected life cycle test within 2 to } \\
3 \text { months }\end{array}$ & Defines Rate \\
\hline 6 & Provide space for optical testing instruments & $\begin{array}{l}\text { 200 mm X 200 mm X 700 mm } \\
\text { optical test bench at input and exit }\end{array}$ \\
\hline 7 & Provide test bed for hexapod control system & Defines Parallel Kinematics \\
\hline
\end{tabular}

\subsection{Test Apparatus Design}

The load-time histories for the IFU test apparatus are generated based on the HET motion. The accelerated life test apparatus consists of two subsystems: the electromechanical hexapod, and the optical bench axis. Early on, segment 2 of the fiber system became the primary concern and the focus of the accelerated life test. It was reasoned that the relative motion between SR1 and SR2 is of greater risk to the fiber system as compared to the motion of segment 3. The length 
of fiber in segment 2 is also shorter than that in segment 3 , resulting in mechanical cycling over a more concentrated segment during the articulation of the fiber bundles. These concerns gave emphasis to calculating the motion between SR1 and SR2 and designing the IFU accelerated life test to closely replicate this motion. The subsystem designed to replicate the motion between SR1 and SR2 is the electromechanical hexapod (see reference 14). The motion of fiber segment three is achieved by translating the optical bench axis in the vertical direction. Space constraints within the test facility prevents an exact replication of fiber segment 3 motion, however, a vertical translation stage driven by a winch provides similar flexing of the fiber bundle.

\section{Motion of fiber system}

For the test, Strain Relief 2 is chosen to be held stationary instead of translating per its operation on the telescope tracker. This allows the motion of fiber segment 2 to be precisely replicated within the available space of the test facility. The relative motion between Strain Relief 1 and Strain Relief 2 was calculated to determine the range of motion required by the electromechanical hexapod. A geometrically driven two dimensional sketch model was used to calculate the movement between SR1 and SR2 given the appropriate telescope parameters.

\section{Design Concepts}

Three design concepts were developed that are capable of achieving the motion between Strain Relief 1 and Strain Relief 2. The methodology used for developing the IFU accelerated life test concepts is expressed through two key points:

- Design for required motion

o Develop and model components that will replicate the required motion

o Utilize minimalistic approach

- Envelope design

- Minimize the number of active (powered) components

o Size components to provide adequate coupling with SR1

o Integrate nominally sized motors, drive screws and gear boxes

o Incorporate necessary bearings or rollers at moving interfaces

The three concepts developed were; 1) the rotary fiber tester, 2) cart / cable fiber tester, and 3) the hexapod fiber test apparatus.

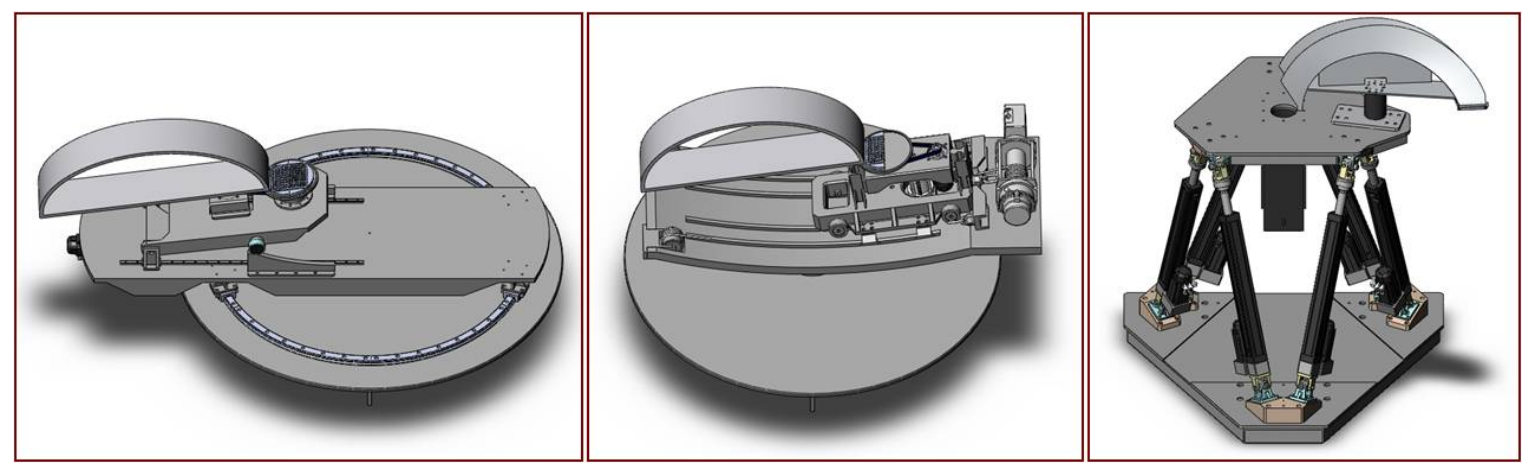

Figure 5. The rotary fiber tester, cart/ cable fiber tester, and the hexapod fiber tester from left to right are the conceptual designs developed for fiber motion between SR1 and SR2.

The hexapod concept was chosen based on evaluation factors such as: ability to replicate movement, form factor, ability to fasten SR1 similarly to the HETDEX design, robustness, design space for optical instruments, fiber damage prevention, and controls testing.

\section{Mechanical Design of Hexapod}

The dynamic behavior of the hexapod fiber test apparatus was modeled using parametric modeling created in SolidWorks software. First the models were used to calculate the actuator design parameters: travel, force and speed. The models were then employed to complete the component design by calculating U-joint displacements and analyzing component stresses. 


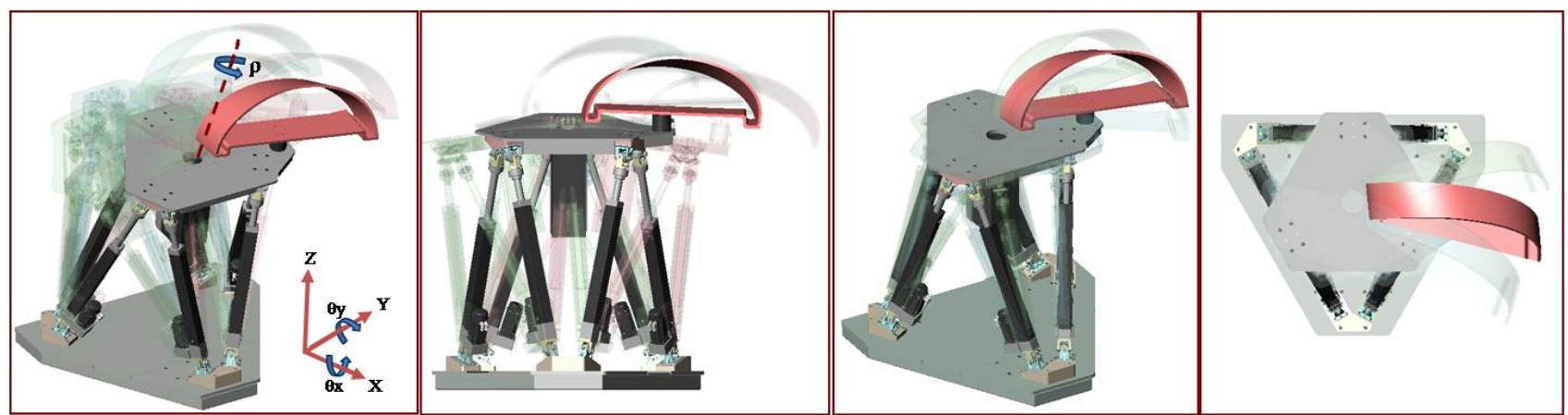

Figure 6. Six DOF motion is achieved by the hexapod test device. The first two images on the left illustrate the motion required to replicate the fiber system motion without rho rotation. Rho rotation is illustrated in the images on the right.

The hexapod model was driven by sketch paths that were derived from geometrically driven two dimensional sketch models. The operational limits for HETDEX occur at tracker X/Y positions of $+/-1870.08 \mathrm{~mm}$ from the center of the primary mirror ${ }^{12,13}$. At this position the focal surface and collector mirror are tilted at $+/-9$ degrees from the tracker plane. The hexapod travel required to explicitly replicate the fiber system movement of segment 2 throughout its operational limits is described by 4 components of motion:

- $\mathrm{Z}=$ Max: $116.33 \mathrm{~mm} \quad$ Min: $0 \mathrm{~mm}$

- $\mathrm{X} / \mathrm{Y}=$ Max: $391.99 \mathrm{~mm}$ Min: $-391.99 \mathrm{~mm}$
- $\theta \mathrm{x} / \theta \mathrm{y}=$ Max: 9 deg Min: $-9 \mathrm{deg}$

- $\rho=$ Max: $21 \mathrm{deg} \quad$ Min: $-21 \mathrm{deg}$

Travel

To determine actuator travel an automated design program positions the hexapod payload platform in the model at over 1500 discrete locations within its travel range. The program accepts a table of payload positions in EXCEL and commands the SolidWorks model to move to each position. At each position the program makes measurements between user specified joints or bodies within the model, records the measurements, and then moves to the next position. Component orientations are also checked at each position to determine if interferences arise. The results from the design tool yield the following:

- Actuator Travel: $496.08 \mathrm{~mm}$

- Upper U-Joint Misalignment: 44 degrees
- Upper U-Joint Rotation: 11 degrees

- $\quad$ Lower U-Joint Misalignment: 31 degrees

Force

The hexapod actuators need enough force capability to position the payload platform throughout the range of motion, and support the cantilevered forces resulting from the fibers suspended from SR1. The mass that hangs from SR1 is approximately $170 \mathrm{~kg}$ which includes 7 meters of conduit and 19 meters of fibers. SolidWorks Simulation was used to perform a system level finite element analysis of the test apparatus to calculate the reaction forces of each hexapod actuator. The maximum reaction force defined the minimum force required by the actuators.

The simulation performed calculations at 20 different payload positions representing different actuator lengths, orientations, and payload locations within the test travel limits. The maximum actuator force calculated was $10.6 \mathrm{kN}$. The 35 degree angle and the orientation of the actuators result in the front two actuators requiring more force than the remaining actuators.

\section{Speed}

The actuator stroke velocity is specified so that the system operates fast enough to complete the 5 year life cycle test of the fibers in 2 to 3 months without introducing inertial effects that are not present during operation on the telescope. The telescope tracker is designed to operate at two different speed ranges; tracking speed and slewing speed. Tracking speed is the speed at which the telescope tracker moves while performing science. Slewing speed is much faster than tracking speed, and is specified for non-tracking moves such as resetting to a different position. By operating the hexapod payload at slew speed the resulting actuator velocity is $21.9 \mathrm{~mm} / \mathrm{s}$. 


\section{Actuator}

The Kollmorgen EC5-B series ball screw actuator was chosen for the six linear actuators required to build the hexapod for the fiber accelerated life test. The EC5 is a Danaher-Motion product operating with a ball screw / ball nut mechanical drive arrangement and a helical gear box that provides additional mechanical advantage. The EC5 actuators are commonly used for industrial automation and are robust linear motion packages that integrate with a variety of motors and drives. The EC5 actuators can operate with the same servo motors and drives that are designed for the telescope tracker system, and thus have the added benefit of providing spare components for the telescope after the test is completed. The ball screw lead, $10 \mathrm{~mm} / \mathrm{rev}$, and helical gear reduction, 10:1, are chosen to prevent over torque and over speed of the AKM53G motor. The resulting performance of the actuator is:

- Torque: $2.4 \mathrm{~N}-\mathrm{m}$ at $11 \mathrm{kN}$ thrust

- $\quad$ Speed: $1,200 \mathrm{rpm}$ at $20 \mathrm{~m} / \mathrm{s}$ linear stroke velocity

The peak mechanical abilities of the motor and actuator are listed below.

Motor:

- $\quad$ Rated Torque: $9.1 \mathrm{~N}-\mathrm{m}$

- $\quad$ Rated Speed: $2400 \mathrm{rpm}$
EC5-B Series Actuator:

- $\quad$ Maximum Thrust: $25 \mathrm{kN}$

- Maximum Speed: $1330 \mathrm{~mm} / \mathrm{s}$

- Maximum Stroke Length: $600 \mathrm{~mm}$

\section{Actuator End Joints}

The hexapod requires a 2 DOF and 3 DOF joint between the actuators and end plates to operate with 6 DOF. Off the shelf components were originally considered but none were found that could achieve the required misalignment. Custom U-joints were designed to handle the large misalignment and axial loads. The 3 degree of freedom joint was chosen to reside on the screw side of the actuator (the upper joint), to limit actuator motion. Risk of power/sensor cable snags and excessive wear of components from mechanical cycling is reduced by attaching the motor side of the actuator to the 2 DOF joint on the stationary platform.

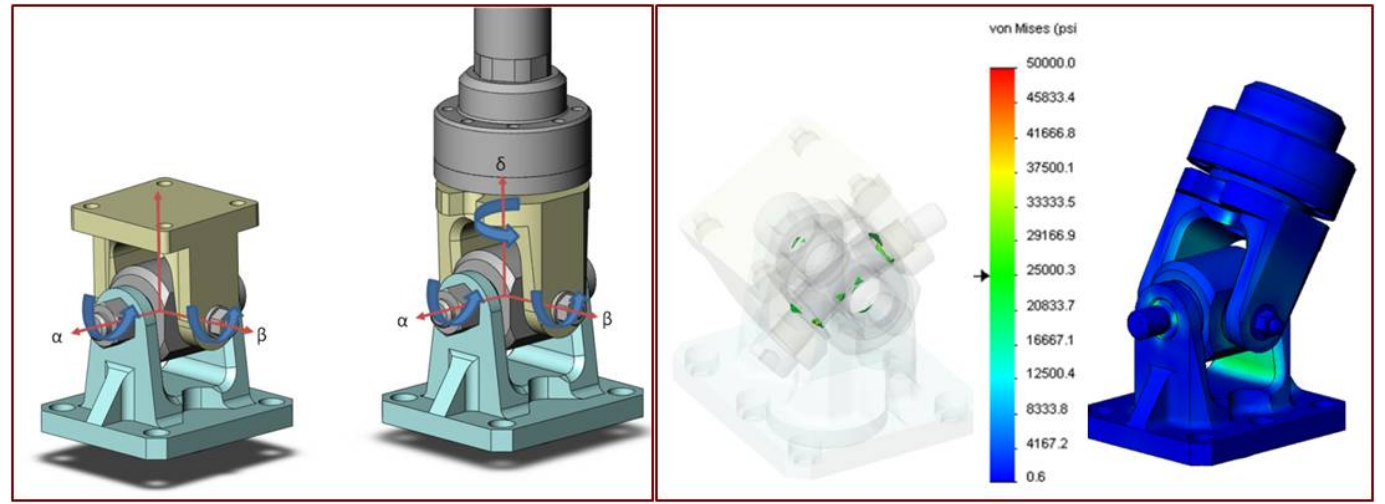

Figure 7. U-joints DOF definitions and analysis images are illustrated above. Both an iso-plot and continuous contour analysis plot are displayed in the far right images respectively.

For the U-joints flanged plain bearings provide the sliding contact between the pins and clevises. Igus, Iglide-Q flanged polymer bearings were chosen for their high load carrying capacity at a very low cost. Needle roller thrust bearings provide rotation about the actuator axis at the 3 DOF joints. Needle roller thrust bearing were chosen because of their compact design, high thrust load carrying capability, and low cost. The bearings were sized by performing calculations from empirically derived equations for bearing life and static load carrying capacity. The predicted life and load carrying capacities of the bearings exceed the required values; the lowest factor of safety is 4.1 for the static load carrying capacity of the Igus Plain Bearing.

Fabricated component stresses were calculated using SolidWorks Simulation. Peak stresses were examined to verify that material limits were not exceeded. The maximum stress calculated by the simulations was 55,000 psi; however mesh convergence could cause the calculated stresses to increase. The simulation results are approximations of component performance that must be validated through experimental verification. Simulation results will be further refined, if 
necessary, by performing mesh convergence studies and developing unique component contact algorithms derived from empirical data. Iso-plots were studied to determine if the high stress magnitudes are contained within small regions. The iso-plots reveal that the high stresses are contained in small regions and that the stresses around those regions quickly tapered to values below $25 \mathrm{ksi}$. The U-joint components were fabricated from normalized AISI 4140 alloy steel that exhibits yield stresses of $100 \mathrm{ksi}$, resulting in a factor of safety of 1.8 above the peak stresses calculated. The base platform and upper platform were fabricated from ASTM A36 and the structural support tubing conforms to ASTM A500B. The base and upper platforms and structural support analyses resulted in a minimum factor of safety of 2.3 against endurance stress.

\subsection{Optical Bench Axis}

The optical bench axis is designed to move the fiber test bundle so that it approximates the dynamic behavior exhibited by fiber segment 3 of the HETDEX fiber system. A wire rope drive is chosen for its simplicity, low cost and adaptability to space constraints and instrumentation integration. The exact motion of fiber segment 3 cannot be replicated due to the space constraints within the laboratory. The change in slope with respect to gravity that occurs in the fiber bundle between the freely hanging bundle and its attachment to SR3, as the tracker moves, is the behavior of concern. The curve formed by the bundle contracts and extends as the telescope tracker moves across the primary mirror. There is concern that the dynamic bend radius that occurs will result in long term effects that affect fiber FRD performance. Measurements were made with a fibrous rope of similar bundle diameter at the Hobby Eberly Telescope to determine the angle of entrance into SR3 and bundle sag as a function of tracker motion. The change in bundle sag is used to determine the length of travel needed for the optical bench axis. The vertical axis which the optical bench is mounted on, guides the fiber bundle so that a dynamic bend radius is created from vertical motion (Figure 8). Fiber segment 4 from SR3 to the VIRUS break out boxes remains static for the HETDEX fiber system (Table 1), therefore the movement of SR3 for the accelerated life test requires that fiber segment 4 for the test bundle remain on the vertically translating platform. The vertical axis is designed to support SR3 and the optical bench and output instruments to achieve a static condition of fiber segment 4.
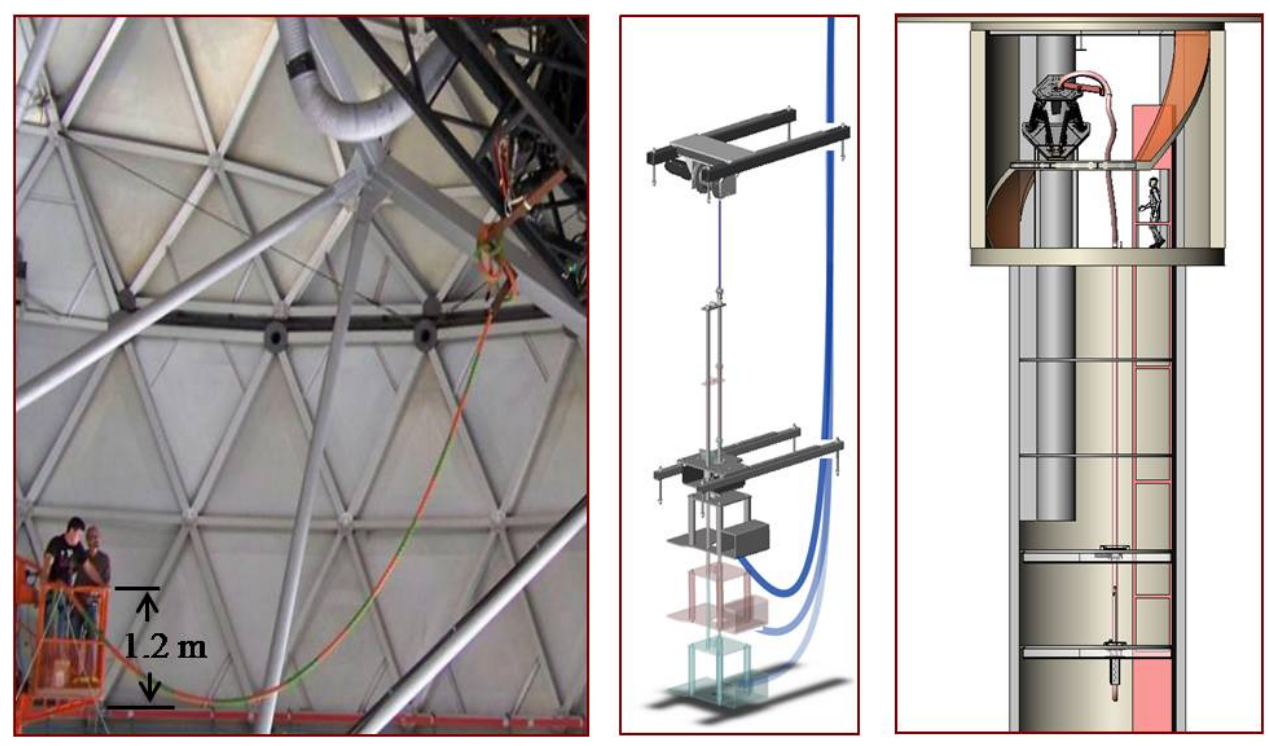

Figure 8. From left to right, measurements at HET, the optical bench axis, and the test layout within the test laboratory.

The mechanical design of the optical bench axis contains two stationary stands and a moving platform. The stationary stands bridge openings in the 4th and 5th floors of the laboratory. The upper stationary stand retains the AKM-53G drive motor and Nema True (40:1) gear box. The AKM-53G is identical to the motors that are used for the hexapod actuators, and the gear box ratio is sized so that the peak motor torque is less than the rated torque of $5.4 \mathrm{~N}-\mathrm{m}$. The vertical winch drive will move $1.2 \mathrm{~m}$ every cycle resulting in a linear speed of $63.17 \mathrm{~mm} / \mathrm{s}$. The rotational speed required by the motor was calculated to be $271.4 \mathrm{rpm}$. The gear box is coupled to a custom wire rope drum that is supported by two welded brackets. The drum shafts are supported by deep groove ball bearings, SKF 6008-2RS1. The ball bearings are rated for 1.05 mill. revolutions while 0.145 million revolutions are required to achieve the 5 year life cycle. A 1/4" diameter $7 \times 19$ 
galvanized wire rope is chosen to spool onto a $175 \mathrm{~mm}$ diameter drum. The wire rope and drum factor of safeties are 6.0 and 2.5 respectively at $1,800 \mathrm{~N}$. The wire rope attaches to a clevis that is fastened to the top of the primary linear shaft.

The lower stationary platform houses four linear bearings and retains a position sensor and two end of travel sensors. The two primary linear bearings are self-aligning linear ball bearings that guide a $1500 \mathrm{~mm}$ hardened precision shaft. The shaft slides axially through the linear ball bearings. Two bearings are needed to resist the moment applied from the off centered masses attached to the moving platform. Basic linear ball bearings are chosen based on their adequate performance characteristics with low cost. To prevent rotation of the lower platform a secondary linear shaft is added. The secondary linear shaft "floats" through a set of oversized linear plain bearings. A floating arrangement is chosen so that precise alignment can be avoided and binding prevented. The plain bearings are Igus, Iglide-J linear plain bearings, chosen for their low cost.

The moving platform is fastened to the bottom of the shafts and supports SR3 and the optical instruments required at the fiber output. The moving platform contains a lower plate that it modular and can be un-bolted and modified as necessary to fasten components and instruments that have not been fully defined.

\section{IFU TESTING CONTROL SYSTEMS DESIGN}

\subsection{Sensors}

Controlling the IFU test system requires moving the hexapod and optics bench in concert. Independent linear sensors in each axis are the primary feedback for the motion controller. Numerous secondary inputs are necessary for fault detection. The sensor suite for the IFU test system is:

- (6) Linear potentiometers in the EC5 actuators from Danaher

- $\quad$ PT510 string potentiometer for the optics bench from Celesco

- (12) PSP-2 limit switches installed on the actuators

- GLC limit switches from Honeywell Corporation. These switches are installed at each end of the optics bench travel range.

- TLL-1K load cells from Transducer Techniques. These load cells are installed in the fiber bundle

- (7) Current feedback from servo-amplifiers

- (7) Speed feedback from servo-amplifiers

- Status of the high-power contactor

- (7) RTO (Ready to operate) status of servo amplifiers

\subsection{Controller Hardware}

\section{dSPACE Controller}

A dSPACE autobox is the controller for the IFU tester. It contains the following hardware:

- DS1005 - Control board

- DS2003 - 32 channel analog in board

- DS2103 - 32 channel analog out board

- DS4003 - 96 channels of discrete I/O

The dSPACE system will operate at speed of $1 \mathrm{kHz}$ to control the IFU test motion.

Data Acquisition System

A mixture of analog and discrete sensors is used in this control system. They all are captured via a data acquisition system composed of the following components:

- (3) OM7-BP-16 - 16 channel analog board

- (2) $1782-\mathrm{A} 16 \mathrm{H}-16$ channel discrete I/O board

Two of the OM7 boards are used for analog inputs and are routed to the DS2003 board. The other OM7 board is used for analog outputs and is routed to the DS2103 board. The discrete boards are both connected to the DS4003 board. 


\section{Motor Drives}

Danaher S603 servo amplifiers were chosen to drive the IFU hexapod and the optics bench axis. These drives have a continuous current rating of 3 amps and a peak of 6 amps. After analysis of the system loads, gear ratios were chosen to assure motor currents would be less than 3 amps. The actuator with the heaviest load will require 2 amps while the others will require less.

The drives will be commanded using analog torque mode. Analog signals transmitted from the dSPACE controller will be scaled to a current command. The drives will then be responsible for closing the current loop in the motors.

\subsection{Controller Algorithm}

\section{Motion Control}

Duplicating the range of motion that the IFUs will see on the tracker with the IFU tester requires all seven axes to work in unison. The hexapod upper frame motion generates linear motion in three linear axes $(\mathrm{X}, \mathrm{Y}, \mathrm{Z})$ and rotational motion about three axes to simulate motions imparted to fiber bundles during actual HET tracking and slewing operations. Specialized algorithms determine required location and orientation of the upper frame with respect to the lower frame (as a function of time), which allows easy determination of required leg-lengths (as a function of time) to achieve desired location/orientation. Once required strut lengths are determined, each actuator will be individually controlled using a tuned PID feedback loop with feed forward force commands to offset static loads due to payload mass. Vertical motion at SR3 of the IFUs is captured by the optical bench axis. The optics bench axis will be controlled similarly with a PID loop with feed forward force commands.

\section{Path Planning}

Accelerated life testing of the IFUs will be performed using a variety of motion paths. To simulate the tracker motion, paths which simulate different speeds and profiles will be programmed into the dSPACE controller. These different profiles will be integrated together to simulate how the tracker will perform with the IFUs.

\subsection{Faults}

Fault monitoring is an important function for the IFU tester controller. The various fault conditions monitored are:

- Over-travel - Both software and limit switches continually monitor for over-travel conditions.

- Over-current - Software continually monitors the motor current. Due to the repetitive nature of the IFU motion, the current required is well defined. If the motor current exceeds this amount then a fault is generated.

- Drive Fault - The RTO signals from the drives are continually monitored.

- $\quad$ Load Cell Fault - The IFU load cells will be monitored for load readings that diverge from their nominal readings.

A fault causes the controller to stop the IFU test motion and engage the brakes.
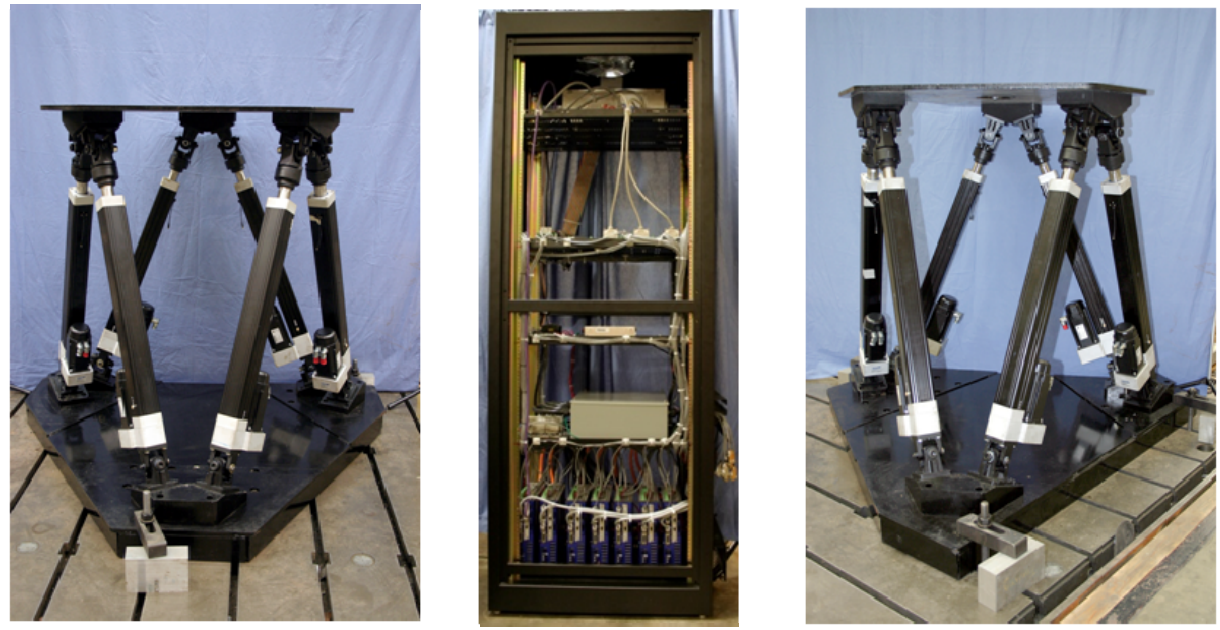

Figure 9. IFU Hexapod and controller cabinet assembled in CEM lab. 


\section{SUMMARY}

The size and complexity of the fiber system for HETDEX is much greater than previous fiber systems deployed at the McDonald Observatory, and elsewhere in the astronomy community. Implementing a mechanical support system for 96 IFUs presents challenges to the HETDEX program. An accelerated life test will soon be underway to study the impacts that repeated cycles of extending, contracting and flexing of the IFUs have on the throughput and longevity of individual fibers. This experiment will be useful in; 1) evaluating the existence of any FRD in individual fibers, 2) studying the bulk behavior and dynamics of 448 fibers inside a conduit, as well as 3) determining the behavior and associated forces required to manipulate and support 96 IFU conduits. Fiber system design refinement and modifications will be implemented if weaknesses are exposed through testing. The effects of accelerated load histories on performance of optical fibers will be of great interest to designers of robotic fiber handling systems for major telescopes.

\section{REFERENCES}

[1] Hill, G. J., Gebhardt, K., Komatsu, E., Drory, N., MacQueen, P. J., Adams, J. J., Blanc, G. A., Koehler, R., Rafal, M., Roth, M. M., Kelz, A., Gronwall, C., Ciardullo, R. and Schneider, D. P., "The Hobby-Eberly Telescope Dark Energy Experiment (HETDEX): Description and Early Pilot Survey Results," ASP Conf. Series, 115-118 (2008) .

[2] Savage, R.D., et al., "Current status of the Hobby-Eberly Telescope wide-field upgrade," Proc. SPIE 7733-149 (2010).

[3] McDonald Observatory, "HETDEX Illuminating the Darkness." [Online]. hetdex.org/hetdex/index.php. (2008, January)

[4] Kelz, A., Roth, M. M., Bauer, S. M., Padilla, Y., Popow, E., Hill, G. J., Vattiat, B. L., Good, J. M., Murphy, J. D., Savage, R. D., Soukup, I. M. and Mollison, N. T., "Production and performance of replicable integral field units for VIRUS," Proc. SPIE 7735-178 (2010).

[5] Hill, G. J., et al., "VIRUS: a massively replicated 33k fiber integral field spectrograph for the upgraded HobbyEberly Telescope," Proc. SPIE 7735-21 (2010).

[6] Good, J. M., et al., "Current Status of the HETDEX Fiber Optic Support System," Proc. SPIE 7014, 70147L-1 (2008).

[7] Murphy, J. D., MacQueen, P. J., Hill, G. J., Grupp, F., Kelz, A., Palunas, P., Roth, M. and Fry, A., "Focal Ratio Degradation and Transmission in VIRUS-P Optical Fibers," Proc. SPIE 7018, 70182T-1 (2008)

[8] Parry, R. I., "The Astronomical uses of Optical Fibers," Proc. Fiber Optics in Astronomy III, ASP Conference Series 152 (1998).

[9] Ramsey, L. W., "Focal ratio degradation in optical fibers of astronomical interest," Proc. Fiber Optics in Astronomy, ASP Conference Series A90-20901 07-35, 26-39 (1988).

[10] Lee, D., Haynes, R. and Skeen, D. J., "Properties of Optical Fibers at Cryogenic Temperatures," MNR Astronomical Society 326, 774-780 (2001).

[11]A. Kelz, et al., "Production and performance of replicable integral field units for VIRUS," Proc. SPIE, 7735-178 (2010).

[12] Worthington, M. S., Beets, T. A., Good, J. M., Mock, J. R., Murphy, B. T., and South, B. J., "Design and development of a high precision, high payload telescope dual drive system," Proc. SPIE 7733, 201 (2010).

[13] Mollison, N. T., et al., "Design and development of a long-travel positioning actuator and tandem constant force actuator safety system for the Hobby-Eberly Telescope wide-field upgrade," Proc. SPIE 7733, 150 (2010).

[14] Mock, J. R., Beno, J. H., Zierer, J. J., Rafferty, T. H., Cornell, M. E., "Tracker controls development and control architecture for the Hobby-Eberly Telescope wide-field upgrade,” Proc. SPIE 7733, 152 (2010)

\section{$\underline{\text { Acknowledgement }}$}

HETDEX is led by the University of Texas at Austin McDonald Observatory and Department of Astronomy with participation from the Universitäts-Sternwarte of the Ludwig-Maximilians-Universität München, the Max-PlanckInstitut für Extraterrestriche-Physik (MPE), Astrophysikalisches Institut Potsdam (AIP), Texas A\&M University, Pennsylvania State University, and the HET consortium. In addition to Institutional support, HETDEX is funded in part by gifts from Harold C. Simmons, Robert and Annie Graham, The Cynthia and George Mitchell Foundation, Louis and Julia Beecherl, Jim and Charlotte Finley, Bill and Bettye Nowlin, Robert and Fallon Vaughn, Eric Stumberg, and many others, by AFRL under agreement number FA9451-04-2-0355, and by the Texas Norman Hackerman Advanced Research Program under grants 003658-0005-2006 and 003658-0295-2007. 